\title{
Circulating microRNAs as biomarkers for rituximab therapy, in neuromyelitis optica (NMO)
}

Adi Vaknin-Dembinsky ${ }^{1 * \dagger}$, Hanna Charbit $^{1 \dagger}$, Livnat Brill', Oded Abramsky ${ }^{1}$, Devorah Gur-Wahnon², Iddo Z. Ben-Dov ${ }^{2}$ and Iris Lavon ${ }^{1,3}$

\begin{abstract}
Background: Neuromyelitis optica (NMO) is a chronic autoimmune disease of the central nervous system (CNS). The main immunological feature of the disease is the presence of autoantibodies to Aquaporin 4 (AQP4+), identified in about $82 \%$ of cases. Currently, there are no reliable biomarkers for monitoring treatment response in patients with NMO. In an effort to identify biomarkers, we analyzed microRNAs (miRNAs) in the blood of rituximab-treated NMO patients before and after therapy.

Methods: Total RNA extracted from whole blood of nine rituximab-responsive NMO patients before and 6 months following treatment was subjected to small RNAseq analysis. The study included an additional group of seven untreated AQP4+ seropositive NMO patients and 15 healthy controls (HCs).

Results: Fourteen miRNAs were up regulated and 32 were downregulated significantly in the blood of NMO patients following effective therapy with rituximab (all $p<0.05$ ). Furthermore, we show that expression of 17 miRNAs was significantly higher and of 25 miRNAs was significantly lower in untreated NMO patients compared with HCs (all $p<0.05$ ). Following rituximab treatment, the expression levels of 10 of the 17 miRNAs that show increased expression in NMO reverted to the levels seen in HCs. Six of these "normalized" miRNAs are known as brain-specific/enriched miRNAs.

Conclusions: Specific miRNA signatures in whole blood of patients with NMO might serve as biomarkers for therapy response. Furthermore, monitoring the levels of brain-specific/enriched miRNAs in the blood might reflect the degree of disease activity in the CNS of inflammatory demyelinating disorders.
\end{abstract}

Keywords: Brain-specific microRNAs, Circulating biomarkers, Rituximab, Aquaporin 4 (AQP4), Neuromyelitis optica (NMO)

\section{Background}

Neuromyelitis optica (NMO) is a chronic inflammatory disease of the central nervous system (CNS), whose clinical features include mainly acute attacks of bilateral or rapidly sequential optic neuritis (eventually leading to visual loss in many patients), and transverse myelitis. Disease etiology of NMO is still unknown. However, it is known that the

\footnotetext{
* Correspondence: adembinsky@gmail.com

${ }^{\dagger}$ Equal contributors

${ }^{1}$ Department of Neurology, Multiple Sclerosis Center and Laboratory of Neuroimmunology, and the Agnes-Ginges Center for Neurogenetics, Hadassah Hebrew University Medical Center, Ein-Karem, Jerusalem 91120, Israel

Full list of author information is available at the end of the article
}

inflammatory processes in $\mathrm{NMO}$ are mediated by the humoral immune system and primarily target astrocytes $[1,2]$. The most important evidence of this was the identification of the NMO-IgG antibody, anti-Aquaporin-4; NMO-IgG antibodies identify about $82 \%$ of patients. At present, it is challenging to diagnose NMO patients that are negative for this marker from patients with other CNS demyelinating diseases, especially multiple sclerosis (MS).

Micro RNAs (miRNAs) function to modify the expression of target genes. miRNA-mediated gene regulation is critical during many biological processes including inflammation and neurodegeneration. Because each miRNA can regulate many target genes, the biological 
impact of dysregulation of a single miRNA can be considerable. Recent research has shown that miRNAs have potential as non-invasive biomarkers for the diagnosis and prognosis of disease as well as monitoring of treatment response [3]; miRNAs also represent promising novel targets for therapy [1]. Extracellular circulating miRNAs are remarkably stable [4]. Their stability is achieved via different mechanisms. They can be packaged in microparticles (exosomes, microvesicles, and apoptotic bodies) [5-7] or associated with RNA-binding proteins (Argonaute2 [Ago2]) or lipoprotein complexes (highdensity lipoprotein [HDL]) [8] to prevent their degradation. The stability of miRNAs, coupled with advances in high-throughput technologies that provide the ability to perform a global analysis of miRNA expression profiling, has positioned miRNAs as ideal biomarker candidates. Recently, altered miRNA expression has been reported in several human autoimmune diseases, and miR-92a was suggested as a circulating biomarker for disease staging in MS. The single study on miRNAs in NMO revealed that several miRNAs have distinct expression levels in NMO patients compared with healthy controls (HCs) and patients with MS [9]. But, additional more comprehensive studies are needed to establish these miRNAs as diagnostic markers.

Currently, the most commonly used treatments in NMO are steroids, azathioprine, mycophenolate mofetil, and rituximab [10-13]. Rituximab is a chimeric anti-CD20 monoclonal antibody that depletes B cells. It is commonly used for treating B cell lymphoma and has been found to be effective in the treatment of autoimmune rheumatological and neurological conditions including NMO. Rituximab is currently considered the most effective therapy for preventing NMO exacerbations [14, 15]. No definite biomarker of response to therapy is presently available for monitoring patients with NMO. Current data relating to the correlation between AQP4-IgG titers and disease activity in the long-term course of NMO are inconsistent [16].

In this study, we aimed to identify miRNA biomarkers in the peripheral blood of patients with $\mathrm{NMO}$ and to monitor treatment response. We examined whether analysis of global miRNA expression in the blood of patients with NMO before and after treatment with rituximab could identify a distinct miRNA expression signature associated with therapy. This putative signature could serve as a predictor for response to therapy, with the goal of assisting in the individualized management of this disease.

\section{Methods}

\section{Subjects}

The patient cohort included 16 patients with NMO (14 females, two males; age $41 \pm 14.3$ years; disease duration, $5.3 \pm 6.2$ years; Expanded Disability Status Scale (EDSS), $4.8 \pm 1.8$ ), followed at the Hadassah MS Center. The relapse rate in the 2 years prior to the study was $1.25 \pm$ 0.7. None of the enrolled patients had relapse or were treated with steroids for at least 30 days before their blood samples were drawn. $82.3 \%$ of the NMO patients were positive for anti-AQP4. Brain magnetic resonance imaging (MRI) was normal or compatible with the diagnosis of NMO in all patients, and spinal MRIs revealed long extensive myelitis in $14 / 16$ patients. None of the nine responders had relapses in the 2 years following rituximab therapy. Five patients with treatment failure had comparable clinical characteristics to the responders but experienced no decrease in relapse rate with rituximab therapy (four females, one male; age $46 \pm 10.7$ years; disease duration, $5.2 \pm 4.0$ years; EDSS, $5.2 \pm 4.4$; relapse rate in the 2 years prior to the study, $1.3 \pm 0.44$ ). The patients signed informed consent. Clinical data were collected from patients' files. NMO patients were diagnosed according to the NMO diagnostic criteria [17]. An age- and sex-matched control group comprised 15 healthy individuals (10 females, 5 males; age $36.7 \pm 9.4$ years).

NMO-Ig seropositivity in the study cohort was determined using RSR ELISA assay in sera. The anti-AQP4 ELISA positive samples were also assessed using a cellbased assay (Euroimmun).

\section{Blood RNA isolation and miRNA quantification}

A 4-ml blood sample was collected in EDTA tubes from each patient. Two hundred fifty microliters of whole blood was mixed with $750 \mu \mathrm{l}$ of Tri-reagent BD (Sigma), supplemented with $20 \mu \mathrm{l}$ of $5 \mathrm{~N}$ acetic acid, and frozen. RNA was extracted according to the manufacturer's instructions. Gel electrophoresis confirmed the integrity of the RNA, and total RNA was quantified using Qubit 2.0 (Thermo Fisher Scientific Inc.).

\section{Small RNA sequencing}

Total RNA was subjected to multiplexed small RNA cDNA library preparation. Library preparation entails ligation of barcoded 3 ' adapters to 20 different samples, pooling of samples, ligation of a $5^{\prime}$ adapter, reverse transcription and polymerase chain reaction (PCR), as previously described [18], with modifications allowing multiplexing of several 20-sample libraries on a single HiSeq lane, namely, 40-100 small RNA libraries per lane. Libraries were sequenced on an Illumina HiSeq sequencer, and the information obtained was analyzed by an automated computer pipeline to decode and annotate small RNA reads [19]. Normalization of miRNA reads was performed by dividing each miRNA read frequency by the total number of miRNA sequence reads within the subsample, thereby correcting the variable sequencing depth in each subsample. 


\section{Real-time PCR}

Mature miRNAs were quantified using Perfecta ${ }^{\circ}$ microRNA Assays (Quanta Biosciences), according to the manufacturer's instructions. Real-time polymerase chain reaction (PCR) was performed on a StepOne real-time reverse transcription (RT)-PCR (Life Technologies) in triplicate for each sample. The fold changes of miRNAs were normalized to RNU6B, $(\triangle \mathrm{CT})$. The data is presented as $2^{-\Delta \mathrm{CT}}$. Primer sequences were designed based on the miRNA sequences obtained from the miRBase database (http://microrna. sanger.ac.uk/). Statistical significance was calculated using two-tailed $t$ test.

\section{Statistical analysis}

Statistical procedures on count data were based on DESeq2, a publically available R/Bioconductor package for analysis of differential expression in RNA sequencing experiments [20], as previously described [21].

\section{Results \\ Identification of differentially expressed microRNAs before and after rituximab treatment and between HCs and patients with NMO}

The main aim of our study was to identify miRNAs that are differentially expressed before and after rituximab treatment. Rituximab is the most effective therapy for patients with NMO, with a response rate of approximately $90 \%$ [22, 23]. Blood samples were collected from nine NMO patients, classified as rituximab responders, prior to and at 6 months following therapy and from 15 age- and sex-matched HCs. To further analyze the differential expression between untreated NMOs and HCs, seven additional untreated seropositive NMO patients were also included in the study. Total RNA was extracted from the whole blood samples, and miRNA levels were quantified by deep sequencing analysis (RNAseq).

RNAseq results demonstrated that the expression levels of 32 miRNAs were decreased and of 14 miRNAs were increased significantly $(p<0.05)$ in the nine treated NMO patients following treatment with rituximab (Table 1). These nine samples from responders were further compared with those from five non-responders. Using real-time RT-PCR, we were able to show that miR-125 was significantly different for rituximab responders and nonresponders $(p=0.03)$. The additional eight miRNAs that were analyzed were differently expressed in nonresponders, but the differences were not statistically significant, most likely due to the small number of nonresponders in our cohort. (Data from the three most significant miRNAs are presented in Additional file 1: Figure S1).

Analysis of the differential expression between the 16 untreated NMO patients and $15 \mathrm{HCs}$ demonstrated that expression levels were significantly lower for 25 miRNAs and significantly higher for 17 miRNAs in NMO patients compared with HCs (Table 2). Notably, the expression levels for 10 out of the 17 miRNAs with significantly increased expression in NMO patients reverted to the level of HCs following therapy with rituximab (Table 1, Fig. 1a). In addition, miR-7 and miR-124, which had lower expression in NMO patients, reverted to the level of HCs following therapy with rituximab (Table 1, Fig. 1b).

\section{Discussion}

Our results demonstrate for the first time that a set of miRNAs with elevated expression in the blood of NMO patients revert to the levels of matched healthy controls following effective therapy with rituximab. The majority of these "normalized" miRNAs are known as brain-specific/ enriched miRNAs. Following further validation, the results of this study might lead to the establishment of a diagnostic test to monitor treatment response in NMO.

The six brain-enriched miRNAs, miR-135a, miR-135b, miR-125b, miR-134, miR-138, and miR-760 [24-27], that revert to healthy control levels after treatment are expressed abundantly or predominantly in the brain. The higher quantity of these miRNAs in the circulation of patients with NMO might imply that they were shed to the circulation by degenerative brain cells, with a corresponding decrease in the amount of these miRNAs in the circulation observed following treatment, when the destructive inflammatory process diminishes. Therefore, monitoring the levels of brain-specific/enriched miRNAs in the blood might reflect the degree of disease activity in the CNS of demyelinating disorders.

miRNAs are highly stable elements that can be detected in body fluids as well as in different blood cells [28]. Their stability, along with the recent development of sensitive detection and quantification methods, has positioned miRNAs as great candidate biomarkers for diagnosis of specific diseases. Moreover, miRNAs might play a major role in initiating and/or maintaining autoimmunity processes $[29,30]$.

For many years, NMO was considered as a severe variant of MS [1]. Both diseases have autoimmune inflammatory lesions in the CNS. In MS, there is an abundance of data on miRNA expression in whole blood, peripheral blood mononuclear cells (PBMCs), plasma, cerebrospinal fluid (CSF), and peripheral blood $\mathrm{T}$ cells [31-34]. It is widely accepted that miRNAs are dysregulated in MS, but their use as biomarkers is still evolving [31]. In a recently published review on the available data regarding miRNAs in MS, Xinting et al. summarized that miR-15a, miR-19a, miR-22, miR-210, and miR-223 were upregulated in T-reg cells, plasma, blood cells, PBMCs, and brain white matter tissues from MS patients; miR-21, miR-142-3p, miR-146a, miR-146b, miR-155, and miR-326 were upregulated and miR-181c and miR-328 were downregulated in PBMCs 
Table 1 Differential expression of miRNAs following rituximab therapy

\begin{tabular}{|c|c|c|c|c|c|}
\hline \multicolumn{3}{|l|}{ Upregulated miRNAs } & \multicolumn{3}{|l|}{ Downregulated miRNAs } \\
\hline miRNA treated vs untreated & Fold change & $p$ value & miRNA treated vs untreated & Fold change & $p$ value \\
\hline hsa-miR-16 & 1.99 & 0.00001 & hsa-miR-125b $(\# \wedge)$ & 0.17 & 0.00001 \\
\hline hsa-miR-15a & 2.65 & 0.00001 & hsa-miR-760 $(\# \wedge)$ & 0.08 & 0.00005 \\
\hline hsa-miR-124 & 6.42 & 0.00012 & hsa-miR-135a $(\# \wedge)$ & 0.18 & 0.00058 \\
\hline hsa-miR-26b & 1.91 & 0.00044 & hsa-miR-134 $(\# \wedge)$ & 0.1 & 0.00183 \\
\hline hsa-miR-7 & 3.8 & 0.00085 & hsa-miR-138 (\#^) & 0.18 & 0.00515 \\
\hline hsa-miR-15b & 1.79 & 0.00206 & hsa-miR-423-3p (\#) & 0.4 & 0.00734 \\
\hline hsa-miR-451 & 1.85 & 0.00233 & hsa-miR-100 (\#) & 0.31 & 0.00818 \\
\hline hsa-miR-454 & 2.09 & 0.00458 & hsa-miR-125a & 0.24 & 0.00925 \\
\hline hsa-miR-17 & 1.66 & 0.00735 & hsa-miR-135b(\#^) & 0.18 & 0.01455 \\
\hline hsa-miR-144 & 2.13 & 0.01293 & hsa-miR-203 (\#) & 0.2 & 0.01629 \\
\hline hsa-miR-1180 & 3.53 & 0.01837 & hsa-miR-660 (\#) & 0.31 & 0.04582 \\
\hline hsa-miR-106a & 1.71 & 0.01897 & hsa-miR-30d & 0.34 & 0.000002 \\
\hline hsa-miR-184 & 3.41 & 0.03243 & hsa-miR-200c & 0.09 & 0.00003 \\
\hline \multirow[t]{19}{*}{ hsa-miR-374b } & 1.62 & 0.03371 & hsa-miR-30a & 0.18 & 0.00003 \\
\hline & & & hsa-miR-99a & 0.1 & 0.00009 \\
\hline & & & hsa-miR-29a & 0.25 & 0.00034 \\
\hline & & & hsa-miR-200b & 0.1 & 0.00043 \\
\hline & & & hsa-miR-24 & 0.47 & 0.00076 \\
\hline & & & hsa-miR-30e & 0.33 & 0.001 \\
\hline & & & hsa-miR-27b & 0.38 & 0.00199 \\
\hline & & & hsa-let-7c & 0.44 & 0.00711 \\
\hline & & & hsa-miR-375 & 0.14 & 0.00836 \\
\hline & & & hsa-miR-152 & 0.23 & 0.01717 \\
\hline & & & hsa-miR-148b & 0.43 & 0.01862 \\
\hline & & & hsa-miR-361-5p & 0.23 & 0.01937 \\
\hline & & & hsa-miR-221 & 0.46 & 0.02188 \\
\hline & & & hsa-miR-200a & 0.21 & 0.02416 \\
\hline & & & hsa-miR-328 & 0.4 & 0.02717 \\
\hline & & & hsa-miR-92a & 0.71 & 0.02719 \\
\hline & & & hsa-miR-27a & 0.48 & 0.0307 \\
\hline & & & hsa-miR-181a & 0.49 & 0.04338 \\
\hline & & & hsa-miR-143 & 0.38 & 0.04993 \\
\hline
\end{tabular}

miRNAs with increased expression in NMO patients that were normalized following rituximab therapy are indicated by (\#); brain-specific/enriched miRNAs are marked with $(\wedge)$

and brain lesions; and miR-15a and miR-15b were downregulated in blood, peripheral $\mathrm{T}$ cells and $\mathrm{B}$ cells, or plasma samples from MS patients. None of the upregulated miRNAs were increased in our study; the distinct miRNA expression profiles provide further support for the concept that NMO has a distinct pathogenesis from MS.

In MS, there have been limited attempts to study the use of miRNAs in treated versus untreated patients, evaluating commonly used therapies. Waschbisch et al. studied five miRNAs (miR-20b, miR-142-3p, miR-146a, miR-155, and miR-326) by qPCR and found that there was significantly lower expression of miR-142-3p and miR-146a in glatiramer acetate (GA)-treated patients (no differences in expression were detected between untreated and IFN- $\beta$-treated patients) [35]. In an investigation of miRNA and mRNA expression in PBMCs of MS patients before and after IFN- $\beta$ therapy, Hecker et al. found that IFN- $\beta$-responsive genes were upregulated in parallel to downregulation of miRNAs. Among the miRNAs, they identified altered expression among members of the miR-29 family [36]. Given the low clinical response rate of MS patients to IFN $-\beta$ and GA therapy, 
Table 2 Differential expression of miRNAs between untreated NMO patients and HCs

\begin{tabular}{|c|c|c|c|c|c|}
\hline \multicolumn{3}{|l|}{ Upregulated miRNAs } & \multicolumn{3}{|l|}{ Downregulated miRNAs } \\
\hline miRNAs NMO vs HCs & Fold change & $p$ value & miRNAs NMO vs HCs & Fold change & $p$ value \\
\hline hsa-miR-760 (\#^) & 10.6 & 0.00021 & hsa-miR-7 & 0.19 & 0.00034 \\
\hline hsa-miR-423-3p (\#) & 1.6 & 0.0008 & hsa-miR-140 & 0.4 & 0.00042 \\
\hline hsa-miR-660 (\#) & 6.1 & 0.00118 & hsa-miR-22 & 0.57 & 0.00108 \\
\hline hsa-miR-135a (\#^) & 5.3 & 0.00202 & hsa-miR-143 & 0.11 & 0.00109 \\
\hline hsa-miR-135b (\#^) & 8 & 0.00329 & hsa-miR-150 & 0.35 & 0.00126 \\
\hline hsa-miR-1180 & 2.8 & 0.00562 & hsa-let-7b & 0.51 & 0.00221 \\
\hline hsa-miR-203 (\#) & 6.5 & 0.00862 & hsa-miR-2110 & 0.14 & 0.00541 \\
\hline hsa-miR-18a & 1.7 & 0.01101 & hsa-miR-29a & 0.28 & 0.00612 \\
\hline hsa-miR-20b & 1.9 & 0.01591 & hsa-miR-342 & 0.24 & 0.0103 \\
\hline hsa-miR-100 (\#) & 2.8 & 0.02 & hsa-miR-10b & 0.21 & 0.01041 \\
\hline hsa-miR-205 & 6.5 & 0.0228 & hsa-miR-1227 & 0.12 & 0.01501 \\
\hline hsa-miR-134 (\#^) & 4.6 & 0.02493 & hsa-miR-21 & 0.54 & 0.01947 \\
\hline hsa-miR-542 & 6.5 & 0.03172 & hsa-miR-133b & 0.16 & 0.01964 \\
\hline hsa-miR-125b (\#^) & 1.7 & 0.0413 & hsa-miR-532-5p & 0.33 & 0.02214 \\
\hline hsa-miR-551b & 5.7 & 0.04761 & hsa-miR-30e & 0.47 & 0.03266 \\
\hline hsa-miR-454 & 2.1 & 0.04839 & hsa-miR-133a & 0.24 & 0.03284 \\
\hline \multirow[t]{9}{*}{ hsa-miR-138 (\#^) } & 3.5 & 0.04892 & hsa-miR-139 & 0.23 & 0.04032 \\
\hline & & & hsa-miR-124 & 0.33 & 0.0424 \\
\hline & & & hsa-miR-130b & 0.44 & 0.04353 \\
\hline & & & hsa-miR-378 & 0.48 & 0.0439 \\
\hline & & & hsa-miR-215 & 0.28 & 0.04642 \\
\hline & & & hsa-miR-411 & 0.19 & 0.04853 \\
\hline & & & hsa-miR-30a & 0.32 & 0.04876 \\
\hline & & & hsa-miR-27a & 0.46 & 0.04906 \\
\hline & & & hsa-miR-107 & 0.7 & 0.05042 \\
\hline
\end{tabular}

miRNAs with increased expression in NMO patients that were normalized following rituximab therapy are indicated by (\#); brain-specific/enriched miRNAs are marked with $(\wedge)$

the implications of these studies are unclear. For the more effective MS therapy, natalizumab, Sievers et al. found that 10 miRNAs, out of 1059 tested, were differentially expressed in B cells of natalizumab-treated patients versus untreated patients and Ingwersen et al. found that natalizumab therapy restored aberrant blood miRNA expression profiles in MS patients [37, 38]. The 10 most strongly upregulated miRNAs from MS lesions were expressed in astrocytes. This is noteworthy since in NMO the autoantibodies that are directed against AQP4 are expressed by astrocytes. This supports the rationale that miRNAs may contribute to pathogenesis of diseases in which astrocytes play a major role, like NMO.

In contrast to MS, in NMO, there is only one recent publication by Keller et al., which studied miRNAs in the blood of patients [9]. Their study included 11 patients with NMO, 60 patients with MS, and 43 HCs. They identified 141 differentially expressed miRNAs in
NMO patients compared with HCs and 115 miRNAs in NMO versus MS patients. These results did not concur with the results from our study; not all the miRNAs that were found by Keller et al. were detected in our study and vice versa. The difference between the results of these studies may be partly attributed to the differences in patient cohorts. Our study included only untreated patients while most of the patients in the Keller et al. study were treated with immunosuppressive medications. Given the known influence of the immune system on miRNAs [39], it is conceivable that miRNA expression profiles would differ in immunosuppressant-treated and untreated patients. The use of different biostatistical method analyses and different RNAseq library preparation methods might also contribute to the different results [40]. Interestingly, one of the two RT-PCR-validated miRNAs identified by Keller et al. [9] was also highly significant in our cohort-miR-1180 $(p=0.005)$. Although our study 


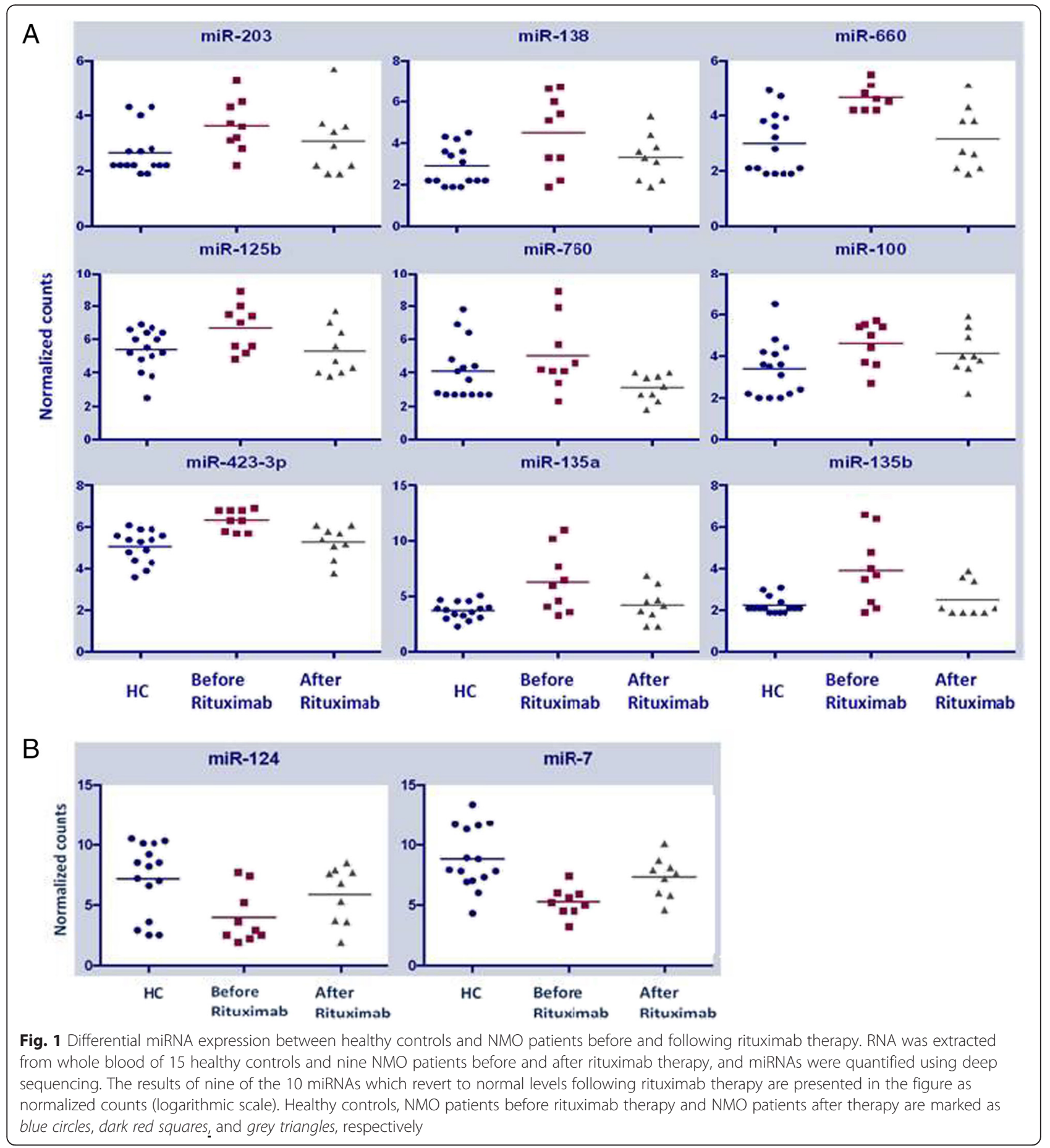

demonstrated differentially expressed miRNAs in NMO patients and HCs, the main focus of our study was on differential miRNA expression after treatment. Using this analysis, we found a clear signature of miRNA expression that not only differed between NMO patients and HCs but also reverted to normal in response to rituximab therapy. Our study has its limitations and currently we cannot ascertain whether some of the above described changes in the
miRNA levels following rituximab therapy are secondary to B cell depletion. It is noteworthy that the blood samples taken at the 6-month time-point were taken following analysis of positive CD19 cell detection in the blood, indicating that patients were not depleted of B cells. This supports the idea that the miRNA signature could represent a distinct phenomenon, rather than simply being a reflection of decreased B cell counts. 
Rituximab is probably the most effective and commonly used therapy in NMO. Although most patients with $\mathrm{NMO}$ have detectable anti-AQP4 autoantibodies in the serum, the mechanism by which $B$ cell-depleting activity mediates the beneficial effect is not clearly understood. Previous studies testing rituximab in NMO found a high response rate and most of the patients remained relapse free or experienced decreases in the annualized relapse rate (ARR) $(60-100 \%$ of disease-free patients in cohorts with 5-30 NMO patients) [22, 23].

Using 18 samples obtained from the nine responding patients in our study (two from each participant), we identified 46 miRNAs that significantly changed following rituximab therapy $(p<0.05)$ (Table 1$)$. One of the significantly changed miRNA is miR-125. This miRNA has previously been described as a biomarker of rituximab therapy in B cell lymphoma and rheumatoid arthritis (RA) [41]. These findings give further validation to the authenticity of our analysis and suggest that the effect of rituximab on miR-125 levels may not be disease-specific.

Several attempts have been made to develop biomarkers in order to individualize rituximab therapy in NMO patients. CD19+ and CD27 + CD19 + B cell counts were studied by Kim et al. in NMO patients treated with rituximab [22, 42]. CD19 is expressed on B cells and CD27 on memory B cells. They reported that monitoring CD19- and CD27-positive B cell counts in the blood could help dosing the next rituximab administration. However, this assay of CD27-positive B cell suppression by rituximab is not in widespread clinical use. Recently, the same group found that, like in patients with nonHodgkin lymphoma and RA [43], NMO patients carrying the V158F allele of the fragment c gamma receptor 3A (FCGR3A-F) have increased likelihood of incomplete $B$ cell depletion and fivefold increase chance of relapse following rituximab treatment. This effect was more significant in patients that were not treated previously with methotrexate [43].

\section{Conclusions}

In conclusion, we identified a miRNA signature in whole blood of patients with NMO. Following rituximab therapy, the expression levels of the majority of miRNAs $(10 / 17)$ that have higher expression in patients with NMO versus HCs revert to normal levels following rituximab therapy. Our findings imply that this miRNA signature may serve as a biomarker for therapy response. Furthermore, six out of these "normalized" miRNAs are brain-enriched miRNAs. Monitoring the levels of brain-specific miRNAs in the blood of patients with inflammatory demyelinating disorders may reflect the degree of disease activity in the CNS.

\section{Additional files}

Additional file 1: Figure S1. RNA was extracted from whole blood of nine responders and five non-responders NMO patients 6 months following Rituximab therapy and miRNAs were quantified using real-time RT PCR. The results of 3 most significant differentially expressed miRNAs are presented in the figure as $2^{\wedge} \mathrm{ct}$. Responders are marked in dark red and non-responders in grey. (DOCX $32 \mathrm{~kb})$

Additional file 2: Table S1. miRNA normalized counts in patients with NMO. (XLSX 157 kb)

\section{Abbreviations}

AQP4, aquaporin 4; ARR, annualized relapse rate; CNS, central nervous system; CSF, cerebrospinal fluid; EDSS, expanded disability status scale; FCGR3A-F, fragment c gamma receptor 3A; GA, glatiramer acetate; HC, healthy controls; HDL, high-density lipoprotein; miRNAs, micro RNAs; MRI, magnetic resonance imaging; MS, multiple sclerosis; PBMC, peripheral blood mononuclear cells; PCR, polymerase chain reaction; RA, rheumatoid arthritis; $\mathrm{RT}$, reverse transcription

\section{Acknowledgements}

IZB-D is supported by the I-CORE Program of the Planning and Budgeting Committee and The Israel Science Foundation (Grant No. 41/11)

This research was supported by the Nofar Program of the Israel Ministry of Industry, Trade and Labor (Grant No. 50653).

\section{Funding}

IZB-D is supported by the I-CORE Program of the Planning and Budgeting Committee and The Israel Science Foundation (Grant No. 41/11). This research was supported by the Nofar Program of the Israel Ministry of Industry, Trade and Labor (Grant No. 50653).

\section{Availability of data and materials}

The data supporting the conclusions of this article is included within the article file (Additional file 2: Table S1).

\section{Authors' contributions}

$A V$, IL, CS, and LB participated in the design of the study, performed, analyzed, and interpreted the miRNA quantification and RT-PCR, and were major contributors in writing the manuscript. IB and DW performed and analyzed small RNA sequencing. OA was a major contributor in scientifically reviewing the manuscript. All authors read and approved the final manuscript.

\section{Competing interests}

The authors declare that they have no competing interests.

\section{Consent for publication}

Not applicable.

Ethics approval and consent to participate

The research was approved by the ethics committees of Hadassah Medical Center.

\section{Author details}

'Department of Neurology, Multiple Sclerosis Center and Laboratory of Neuroimmunology, and the Agnes-Ginges Center for Neurogenetics, Hadassah Hebrew University Medical Center, Ein-Karem, Jerusalem 91120, Israel. ${ }^{2}$ Nephrology and Hypertension Services, Internal Medicine Wing, Hadassah Hebrew University Medical Center, Ein-Karem, Jerusalem 91120, Israel. ${ }^{3}$ Department of Neurology, the Agnes-Ginges Center for Neurogenetics and Leslie and Michel Gaffin Center for Neuro-Oncology, Hadassah Hebrew University Medical Center, Ein-Karem, Jerusalem 91120, Israel.

Received: 3 March 2016 Accepted: 29 June 2016

Published online: 08 July 2016

\section{References}

1. Wingerchuk DM et al. The spectrum of neuromyelitis optica. Lancet Neurol. 2007;6(9):805-15. 
2. Lennon VA et al. IgG marker of optic-spinal multiple sclerosis binds to the aquaporin-4 water channel. J Exp Med. 2005;202(4):473-7.

3. Sheinerman KS, Umansky SR. Circulating cell-free microRNA as biomarkers for screening, diagnosis and monitoring of neurodegenerative diseases and other neurologic pathologies. Front Cell Neurosci. 2013;7:150.

4. Baumann V, Winkler J. miRNA-based therapies: strategies and delivery platforms for oligonucleotide and non-oligonucleotide agents. Future Med Chem. 2014;6(17):1967-84

5. Zernecke $A$ et al. Delivery of microRNA-126 by apoptotic bodies induces CXCL12-dependent vascular protection. Sci Signal. 2009;2(100):ra81.

6. Valadi $\mathrm{H}$ et al. Exosome-mediated transfer of mRNAs and microRNAs is a novel mechanism of genetic exchange between cells. Nat Cell Biol. 2007;9(6):654-9.

7. Arroyo JD et al. Argonaute2 complexes carry a population of circulating microRNAs independent of vesicles in human plasma. Proc Natl Acad Sci U S A. 2011:108(12):5003-8

8. Vickers $\mathrm{KC}$ et al. MicroRNAs are transported in plasma and delivered to recipient cells by high-density lipoproteins. Nat Cell Biol. 2011:13(4):423-33.

9. Keller A et al. Next-generation sequencing identifies altered whole blood microRNAs in neuromyelitis optica spectrum disorder which may permit discrimination from multiple sclerosis. J Neuroinflammation. 2015;12:196.

10. Trebst $C$ et al. Update on the diagnosis and treatment of neuromyelitis optica: recommendations of the Neuromyelitis Optica Study Group (NEMOS). J Neurol. 2014:261(1):1-16.

11. Kimbrough DJ et al. Treatment of neuromyelitis optica: review and recommendations. Mult Scler Relat Disord. 2012;1 (4):180-7.

12. Papadopoulos MC, Bennett JL, Verkman AS. Treatment of neuromyelitis optica: state-of-the-art and emerging therapies. Nat Rev Neurol. 2014;10(9):493-506.

13. Huh SY et al. Mycophenolate mofetil in the treatment of neuromyelitis optica spectrum disorder. JAMA Neurol. 2014;71(11):1372-8.

14. Jacob A et al. Treatment of neuromyelitis optica with rituximab: retrospective analysis of 25 patients. Arch Neurol. 2008:65(11):1443-8

15. Bedi GS et al. Impact of rituximab on relapse rate and disability in neuromyelitis optica. Mult Scler. 2011;17(10):1225-30.

16. Jarius $S$ et al. Antibody to aquaporin-4 in the long-term course of neuromyelitis optica. Brain. 2008;131(Pt 11):3072-80

17. Wingerchuk DM et al. Revised diagnostic criteria for neuromyelitis optica. Neurology. 2006;66(10):1485-9.

18. Williams $Z$ et al. Comprehensive profiling of circulating microRNA via small RNA sequencing of CDNA libraries reveals biomarker potential and limitations. Proc Natl Acad Sci U S A. 2013:110(11):4255-60.

19. Farazi TA et al. Bioinformatic analysis of barcoded CDNA libraries for small RNA profiling by next-generation sequencing. Methods. 2012;58(2):171-87.

20. Love Ml, Huber W, Anders S. Moderated estimation of fold change and dispersion for RNA-seq data with DESeq2. Genome Biol. 2014;15(12):550.

21. Ben-Dov IZ et al. Cell and microvesicle urine microRNA deep sequencing profiles from healthy individuals: observations with potential impact on biomarker studies. PLoS One. 2016;1 1(1):e0147249.

22. Kim SH et al. A 5-year follow-up of rituximab treatment in patients with neuromyelitis optica spectrum disorder. JAMA Neurol. 2013;70(9):1110-7.

23. Tobin WO, Pittock SJ. Rituximab therapy in neuromyelitis optica: moving toward a personalized medicine approach. JAMA Neurol. 2015;72(9):974-7.

24. Sempere LF et al. Expression profiling of mammalian microRNAs uncovers a subset of brain-expressed microRNAs with possible roles in murine and human neuronal differentiation. Genome Biol. 2004;5(3):R13.

25. Lagos-Quintana M et al. Identification of tissue-specific microRNAs from mouse. Curr Biol. 2002;12(9):735-9.

26. Miska EA et al. Microarray analysis of microRNA expression in the developing mammalian brain. Genome Biol. 2004;5(9):R68.

27. Landgraf $\mathrm{P}$ et al. A mammalian microRNA expression atlas based on small RNA library sequencing. Cell. 2007;129(7):1401-14.

28. Leidinger $P$ et al. The human miRNA repertoire of different blood compounds. BMC Genomics. 2014;15:474.

29. Cubillos-Ruiz JR, et al. Reprogramming immune responses via microRNA modulation. Microrna Diagn Ther. 2013. 1(1)

30. Shen $\mathrm{N}$ et al. MicroRNAs - novel regulators of systemic lupus erythematosus pathogenesis. Nat Rev Rheumatol. 2012;8(12):701-9.

31. Gandhi R. miRNA in multiple sclerosis: search for novel biomarkers. Mult Scler. 2015;21(9):1095-103.

32. Junker $A$ et al. MicroRNA profiling of multiple sclerosis lesions identifies modulators of the regulatory protein CD47. Brain. 2009;132(Pt 12):3342-52.
33. Ma X et al. Expression, regulation and function of microRNAs in multiple sclerosis. Int J Med Sci. 2014;1 1(8):810-8.

34. Keller A et al. Comprehensive analysis of microRNA profiles in multiple sclerosis including next-generation sequencing. Mult Scler. 2014;20(3):295-303.

35. Waschbisch $\mathrm{A}$ et al. Glatiramer acetate treatment normalizes deregulated microRNA expression in relapsing remitting multiple sclerosis. PLoS One. 2011;6(9):e24604.

36. Hecker $\mathrm{M}$ et al. MicroRNA expression changes during interferon-beta treatment in the peripheral blood of multiple sclerosis patients. Int J Mol Sci. 2013;14(8):16087-110.

37. Sievers $C$ et al. Altered microRNA expression in B lymphocytes in multiple sclerosis: towards a better understanding of treatment effects. Clin Immunol. 2012;144(1):70-9.

38. Ingwersen $J$ et al. Natalizumab restores aberrant miRNA expression profile in multiple sclerosis and reveals a critical role for miR-20b. Ann Clin Transl Neurol. 2015;2(1):43-55.

39. Chen CZ et al. Regulation of immune responses and tolerance: the microRNA perspective. Immunol Rev. 2013;253(1):112-28.

40. Head SR et al. Library construction for next-generation sequencing: overviews and challenges. Biotechniques. 2014;56(2):61-4. 66, 68, passim.

41. Duroux-Richard I et al. Circulating miRNA-125b is a potential biomarker predicting response to rituximab in rheumatoid arthritis. Mediators Inflamm. 2014;2014:342524

42. Kim SH et al. Repeated treatment with rituximab based on the assessment of peripheral circulating memory B cells in patients with relapsing neuromyelitis optica over 2 years. Arch Neurol. 2011;68(11):1412-20.

43. Kim SH et al. Treatment outcomes with rituximab in 100 patients with neuromyelitis optica: influence of FCGR3A polymorphisms on the therapeutic response to rituximab. JAMA Neurol. 2015;72(9):989-95.

\section{Submit your next manuscript to BioMed Central and we will help you at every step:}

- We accept pre-submission inquiries

- Our selector tool helps you to find the most relevant journal

- We provide round the clock customer support

- Convenient online submission

- Thorough peer review

- Inclusion in PubMed and all major indexing services

- Maximum visibility for your research

Submit your manuscript at www.biomedcentral.com/submit 